\title{
A PIXEL-DOMAIN POST-PROCESSING TECHNIQUE TO REDUCE THE BLOCKING ARTIFACTS IN TRANSFORM CODED IMAGES
}

\section{Usama Sayed Mohammed}

Electrical Engineering Department, Faculty of Engineering, Assiut University, Assiut, Egypt Email: Usama@aun.edu.eg

(Received November 30, 2010 Accepted January 10, 2011)

The reconstructed images after compression produce annoying blocking artifacts near block boundaries, particularly in a low bit-rate image compression, because each block is transformed and quantized independently. Detection of blocking artifact has an important role in the design of image coding systems. Increasing the bit rate to obtain better quality images is too costly. In this paper, a novel pixel-domain technique for image blocking artifact detection is presented. The algorithm detects the regions of the image which present visible blocking artifacts. The blocking artifact detection and reduction are presented as a post- processing in the image decoder. The algorithm first detects the direction of the artifact through power spectrum estimation of the pixel absolute difference (PAD) in both horizontal and vertical directions. The power spectrum of the PAD is estimated using the Maximum Entropy method (MEM) which is a nonparametric method to perform accurate power spectrum estimation. The reduction process is applied only on the direction of artifacts to improve the image quality. The key of the reduction process is based on the modified-projection operator to give reasonable modifications for the block boundary pixels. The simulation results illustrate the performance of the proposed method for the JPEG decoded images.

KEYWORDS: Blocking Artifacts, compressed image, power spectrum estimation, post-processing, JPEG, image processing.

\section{INTRODUCTION}

Block transform-based image coding is one of the most popular image compression techniques. Since blocks of pixels are treated as single entities and coded separately, correlation among spatially adjacent blocks is not taken into account in coding, which results in block boundaries being visible when the decoded image is reconstructed. Such so-called "blocking" artifacts are often very disturbing, especially when the transform coefficients are subject to coarse quantization.

Generally, in the DCT image compression, the original image is divided into subimages called blocks. Each block is transformed and selected large coefficients are quantized and then stored/transmitted. It is known that the blocking effect is the most significant of all coding artifacts [1-3]. Subjective image quality can be improved by decreasing these blocking artifacts. Many blocking artifact reduction algorithms have 
been proposed [4-6]. However, most of them use the mean squared error (MSE) as the distortion measure. Since the MSE is not good for perceptual image quality assessment [7] and assessing the original images is required, several improved distortion measures have been proposed [8, 9]. In general, the reduction of blocking artifacts can be classified into two approaches. In the first approach, the reduction of blocking artifacts is carried out at the encoding side $[10,11]$, but the methods based on this approach do not conform to the existing standards such as JPEG and MPEG, In the second approach, the reconstructed image is postprocessed aimed at improving its visual quality without any modification in the encoding or decoding mechanisms, making it compatible with the aforesaid coding standards. Because of this advantage, most of the recently proposed techniques follow the second approach and it will be used in this paper. The post-processing techniques have been proposed in different directions. Postfiltering methods for reducing the blocking artifacts are introduced in [12-14]. In these methods, Gaussian filter is used to give the best performance. Ramamurthi and Gersho [2] introduced algorithm based on edge detection. The algorithm distinguishes edge pixels from non-edge pixels and then switches between a one-dimensional and a twodimensional filter accordingly to reduce blocking effects. Some other work is done using adaptive filtering scheme in the post-processing algorithm [15, 16]. There are some approaches to reduce the blocking artifacts based on wavelet representation [1720]. Another direction of research has been introduced to reduce the blocking artifact using the iterative image recovery methods. In these methods, the theory of projections onto convex sets (POCS) is used [1], [21]. The approach to reduce the blocking artifact in the transform domain is introduced in $[22,23]$. The suppression of block to block discontinuities is introduced in the JPEG standard [24]. It uses dc values from current and neighboring blocks for interpolating the first few ac coefficients into the current block. The work introduced in [6] is based on the criterion of mean squared difference of slop (MSDS) [22, 23]. The MSDS is an enhanced form of the mean squared difference, which involves the eight neighboring blocks of the current block. G.A. Triantafyllidis et al. [6] proposed a new algorithm using a novel form of the MSDS by involving the diagonal neighboring pixels. They used the Laplacian statistical model to adopt the DCT coefficients and better estimation of the DCT reconstructed coefficient is introduced. In [29], a post-processing technique in frequency domain is proposed. This technique puts forth a method and an algorithm, working in frequency domain, for the detection and reduction of such blocking artifacts. The artifacts are modeled [29] as 2-D step functions between two neighboring blocks. Presence of the blocking artifacts is detected by using block activity based on human visual system (HVS) and block statistics. The boundary regions between blocks are identified as either smooth or non-smooth regions. The blocking artifacts in smooth regions are removed by modifying a few DCT coefficients appropriately, whilst an edge-preserving smoothing filter is applied to the non-smooth regions. Lee et al. [30] proposed a postprocessing algorithm to reduce the blocking artifacts in JPEG compressed images after classifying them into edge area and monotone area according to the edge map which is obtained after thresholding the gradient absolute image. The signal adaptive filtering consists of a 1-D directional smoothing filtering for edge area and 2-D adaptive average filtering for monotone area. Liu et al. [31] proposed a DCT-domain method for blind measurement of blocking artifacts, by modeling the artifacts as 2-D step functions in shifted blocks. A fast DCT-domain algorithm extracts all the parameters required to 
detect the presence of blocking artifacts, by using HVS properties. Artifacts are then reduced by using an adaptive method. Zeng proposed a simple DCT-domain method for blocking effect reduction, applying a zero masking to the DCT coefficients of some shifted image blocks [32]. However, the loss of edge information caused by the zeromasking scheme is noticeable. Luo and Ward gave a technique, which preserved the edge information [33]. The technique is based on reducing the blocking artifacts in the smooth regions of the image. The correlation between the intensity values of the boundary pixels of two neighboring blocks in the DCT domain is used to distinguish between smooth and non-smooth regions.

In this paper, the proposed algorithm first detects the direction of artifacts through power spectrum estimation of the e pixel absolute difference (PAD) in both horizontal and vertical directions. The power spectrum of the PAD is estimated using the Maximum Entropy method (MEM) which is a nonparametric method to perform accurate power spectrum estimation. The reduction process is applied on the direction of artifacts only to improve the image quality. The key of the reduction process is based on the modified-projection operator to give reasonable modifications to the block boundary pixels. The paper is organized as follows. In section 2, the proposed detection process of blocking artifacts in images is introduced. Section 3 describes the proposed detection process of blocking artifacts in images. Simulation results given in Section 4 evaluate visually and quantitatively the performance of the proposed method. Finally, conclusions are drawn in Section 5.

\section{THE PROPOSED DETECTION PROCESS OF BLOCKING ARTIFACTS IN IMAGE}

Blocking artifact detection process plays an important role in the design of image coding system. In this work, it will be the first step in the decoder to detect the artifacts and consequently improve the reconstructed image quality through the reduction process. Blocking artifact detection and measurement algorithms in $[25,26]$ use the weighted mean-squared difference along block boundaries as the blockiness measure. These techniques cannot distinguish between the real blocking discontinuity and the oscillation of the original image itself.

The proposed detection process is based on the pixel absolute difference (PAD) in both horizontal and vertical direction. For any pixel value $\mathrm{x}$ at position $\mathrm{i}$ the PAD is defined as follows:

$$
P A D(i)=|x(i)-x(i-1)| \quad i=1,2, \ldots . .
$$

First, the (PAD) is calculated in each raw and the power spectrum of the results is then estimated using the Maximum Entropy method (MEM) which is a nonparametric method to perform accurate power spectrum estimation [27, 28]. As to the former problem, it is well known that fast Fourier transform has its own drawbacks in estimating the power spectrum, such as poor resolution owing to the effect of window functions and unrealistic assumptions about extending the data. The MEM is an intuitively satisfying approach to power spectrum density estimation in that a minimal amount of assumptions are made concerning the data. This is in contrast to the classical method which effectively assigns a value of zero to unknown autocorrelation 
values and distorts known values (or estimates) with a window. The MEM works well with all-pole (autoregressive (AR)) model processes and sinusoids.

For an AR sequence of order $\mathrm{p}$, only the autocorrelation estimates, $\operatorname{Rx}(0), \ldots$, $\mathrm{Rx}(\mathrm{p})$ are needed to obtain the AR spectrum. It is important to select an appropriate order $\mathrm{p}$ when using the MEM. Too low an order will yield low resolution while too high an order will result in spurious spectral details. The similar method is used for the column pixel to estimate the power spectrum in vertical direction. Fig. 1 shows the results of the detection process for JPEG compressed Lena image at bit rate of $0.2 \mathrm{bpp}$ and the original Lena image. The original Lena image and the compressed one at 0.2 bpp are shown in Fig. 2 (block size of $8 \times 8$ is used in the encoder).

It is clear in Fig. 1 that the blocking artifact is characterized by the peaks on the power spectrum curve. Moreover, the peaks are noticeable only in raw-wise direction. In this example, the blocking artifact reduction process will be applied on one direction only. Fig. 3 shows the result of the blocking artifact detection process for the JPEG Peppers image at bit rate of $0.2 \mathrm{bpp}$ and the original Peppers image.

Blocking Artifacts in columns

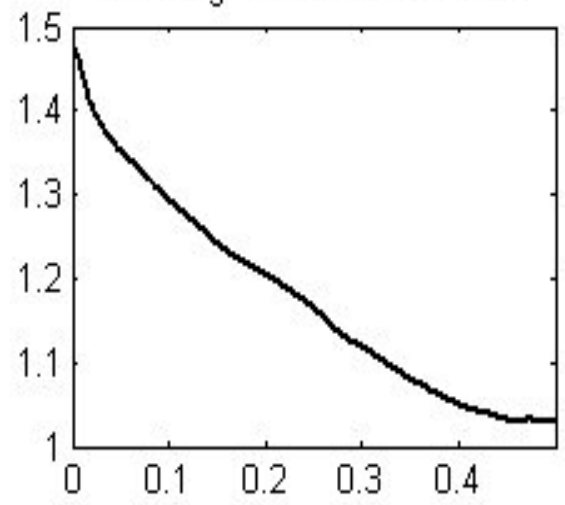

Blocking Artifacts in rows

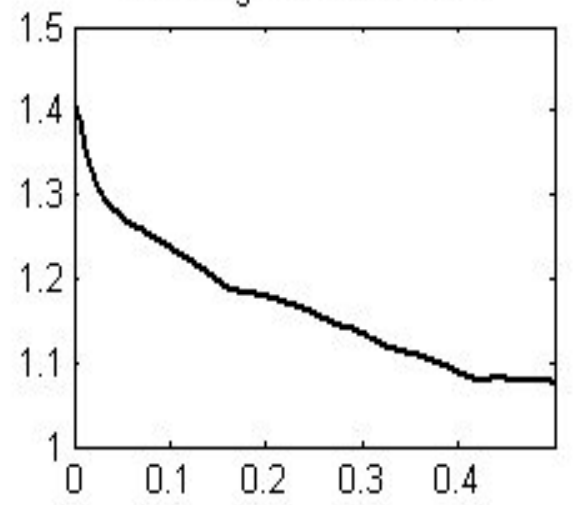

(a) Blocking detection for original Lena image

Blocking Artifacts in columns

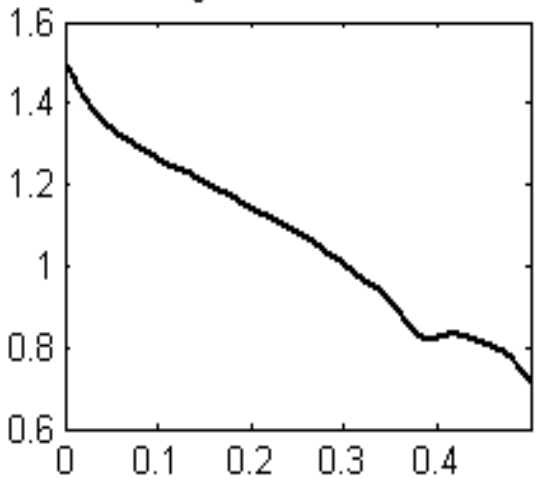

Blocking Artifacts in rows

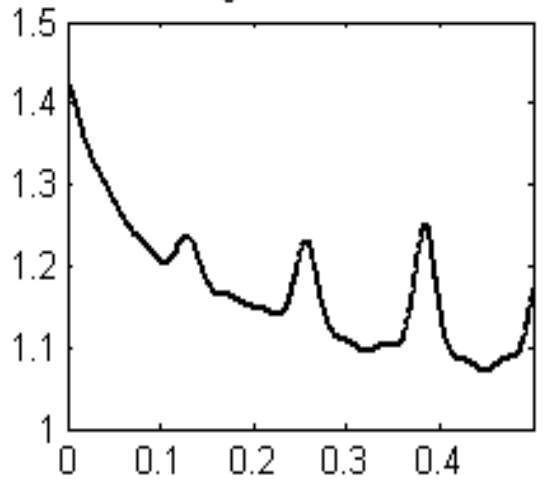

(b) Blocking detection for JPEG compressed Lena image

Fig.1 Power Spectrum of the original Lena image and the JPEG compressed Lena at $0.2 \mathrm{bpp}$. 

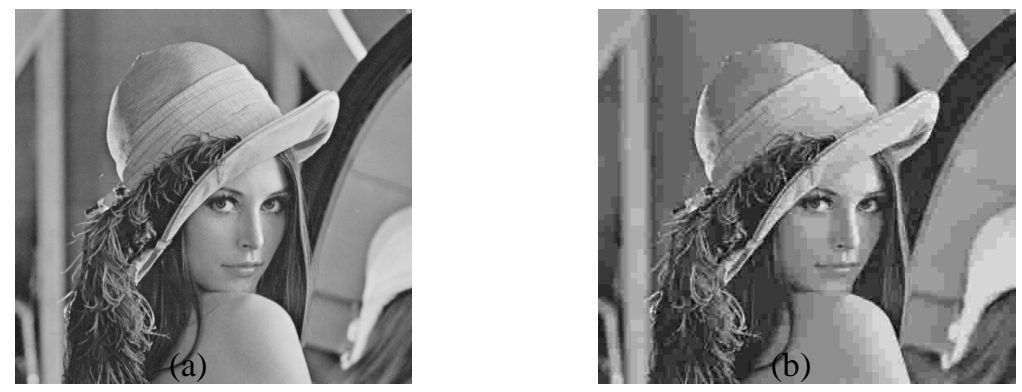

Fig. 2 (a) Original Lena image (b) JPEG compressed Lena image at $0.2 \mathrm{bpp}$.

It is important to note that the Pepper image itself has a non smooth power spectrum distribution, Fig. (3-a), but the peaks in this case are insignificant.

Relative to the result of the detection process the algorithm will be continuing as follows: (1) Terminate the work in the case of no blocking artifact detection. (2) Start the blocking artifact reduction in one direction (horizontal or vertical) or in two dimensions to improve the quality of the decoded image.
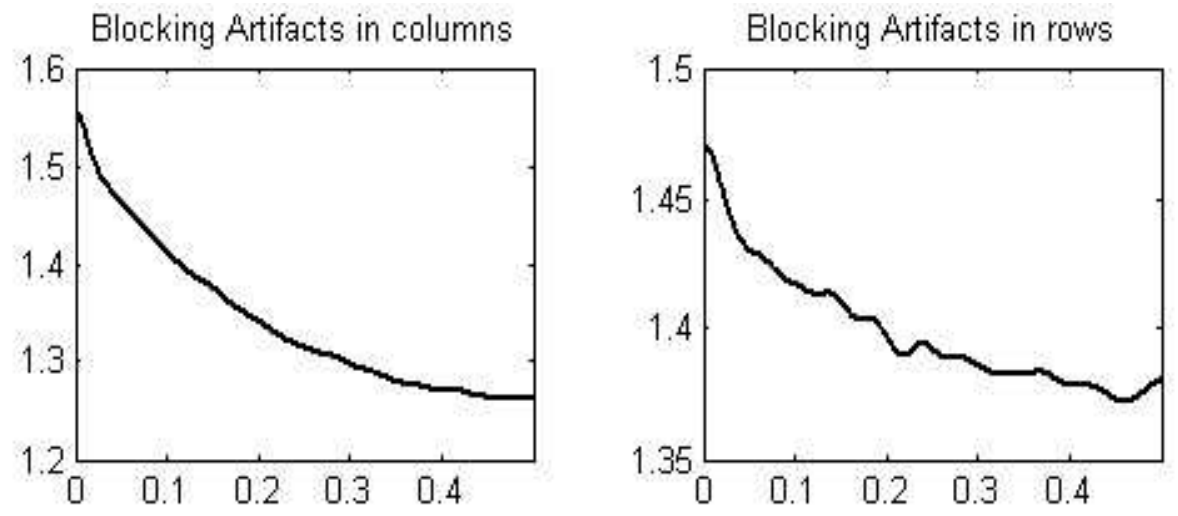

(a) Blocking detection for original Peppers image
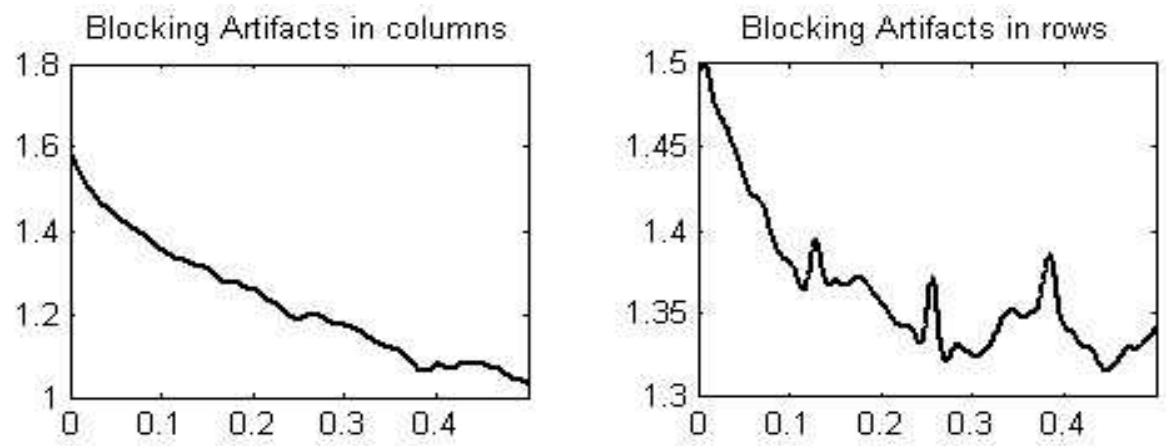

(b) Blocking detection for JPEG compressed Peppers image

Fig.3 Power Spectrum of the original Peppers image and the compressed Peppers at $0.2 \mathrm{bpp}$. 


\section{THE PROPOSED REDUCTION PROCESS OF BLOCKING ARTIFACTS IN IMAGE}

It is known that the blocking noise makes visible grey-level discontinuity at block boundaries. It consists of vertical and/or horizontal false edges which contain significant high frequency components. The proposed blocking artifact reduction approach operates in the pixel domain to reconstruct the distorted boundary pixels from its neighborhoods. The vector of pixels across the boundary of two blocks will be reconstructed based on the best matching vector of pixels from the four surrounding blocks as shown in Fig. 4 for horizontal artifacts.

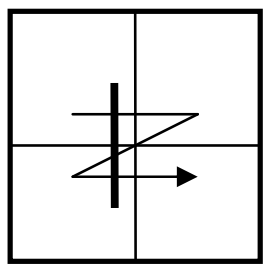

Fig.4 Direction of the matching process.

Let the vector of the pixel values across the boundary of two blocks in the blocky image is $f=(f-j / 2, f-j / 2+1, \ldots, f-1, f 0, \ldots ., f j / 2-2, f j / 2-1)$. Assuming that the image block size is $(i, j)$. The two groups of pixels in the same line of the top blocks and bottom blocks are $\mathrm{f}-\mathrm{j} / 2, \mathrm{f}-\mathrm{j} / 2+1, \ldots \ldots, \mathrm{f}-1$ and $\mathrm{f} 0, \ldots \ldots, \mathrm{fj} / 2-2, \mathrm{fj} / 2-1$, respectively. Assume the vector of pixels within one block, from the four surrounding blocks, is $\mathrm{g}=$ $(\mathrm{g} 0, \ldots \ldots, \mathrm{gj}-2, \mathrm{gj}-1)$. Since the pixels in $\mathrm{g}$ are in the same block, then $\mathrm{g}$ is of good continuity. In the proposed algorithm, the vector $\mathrm{g}$ is used to smooth the $\mathrm{f}$ vector through a nonlinear Laplacian function $\mathrm{y}(\mathrm{x})=\mathrm{A} \exp (-|x|)$, where $\mathrm{A}$ is positive constant. For any vector $\mathrm{f}$ there exist many possible $\mathrm{g}$ vectors within the four surrounding blocks but only one vector from them will be chosen in the projection operation step. The smoothing result is evaluated through the weighted mean-square error (WMSE)

$$
E=\sum_{n=-j / 2}^{j / 2-1} w_{n}\left[f_{n}-y\left(g_{n}\right)\right]^{2}=\sum_{n=-j / 2}^{j / 2-1} w_{n}\left[f_{n}-A e^{-\left|g_{n}\right|}\right]^{2}
$$

where the weight vector $w=(w-j / 2, w-j / 2+1, \ldots ., w-1, w 0, \ldots ., w j / 2-2, w j / 2-1)$ satisfies the following conditions:

$$
\begin{array}{ll}
0 \leq w_{n} \leq 1, & n=-j / 2, \ldots, j / 2-1 \\
w_{-n}=w_{n-1}, & n=1, \ldots, j / 2 \\
\sum_{n=-j / 2}^{j / 2-1} w_{n}=1 &
\end{array}
$$

To obtain the constant value of $\mathrm{A}$ in equation (2), the equation $\partial E / \partial A=0$ must be solved. The constant $\mathrm{A}$ is then equal 


$$
A=\frac{\sum_{n=-j / 2}^{j / 2-1} w_{n} f_{n} e^{-\left|g_{n}\right|}}{\sum_{n=-j / 2}^{j / 2-1} w_{n} e^{-2\left|g_{n}\right|}}
$$

For each vector $\mathrm{g}$ the constant value of $\mathrm{A}$ is evaluated and then the error $\mathrm{E}$ is calculated. One vector of pixels g (at minimum E) will be selected to recover the vector $\mathrm{f}$ through the projection operator [21] as follows:

$$
f_{i}^{\prime}=\alpha f_{i}+(1-\alpha) g_{i}
$$

In the proposed algorithm $\quad \alpha=\frac{1}{2}\left[\frac{E}{\left\|f_{i}-g_{i}\right\|}+1\right]$

The motivation of using the Laplacian function as a matching function is the expected distribution of the slopes formed by the difference of two adjacent vectors in the distorted image.

\section{SIMULATION RESULTS}

The experiments of this work have been applied to the images coded by the JPEG coder standard at different bitrate. The original image is primarily subdivided into a block of pixels with a size of $8 \times 8$ pixels. After blocking detection and reduction algorithms are applied, the PSNR between the decoded image and the original image is calculated. In the simulation results, the weight vector $\mathrm{w}$ is chosen to satisfy equation (3) to equation $(5): \mathrm{w}=(0.2,0.15,0.1,0.05,0.05,0.1,0.15,0.2)$.

The results of the proposed technique for Lena $512 \times 512(8 \mathrm{bpp})$ are shown in Fig.5. It is clear that the proposed method improves the PSNR by about $0.27-0.72 \mathrm{~dB}$. Moreover, the proposed method to reduce the blocking effect will follow the direction of blocking results from the detection process. Fig. 6 gives subjective illustrations of the reconstructed image with and without blocking artifact reduction for Peppers image and Lena image.

The proposed technique achieves nearly the same PSNR as that proposed by [29] whereas the proposed technique gave best results relative to the proposed methods in [30], [31], [33].

Table 1 shows that proposed technique gives best/or same PSNR values as compared to all three methods for different images. It is well known that the PSNR is not always a good measure to reflect the subjective image quality, even though it is one of the most popular criteria employed in image processing. 


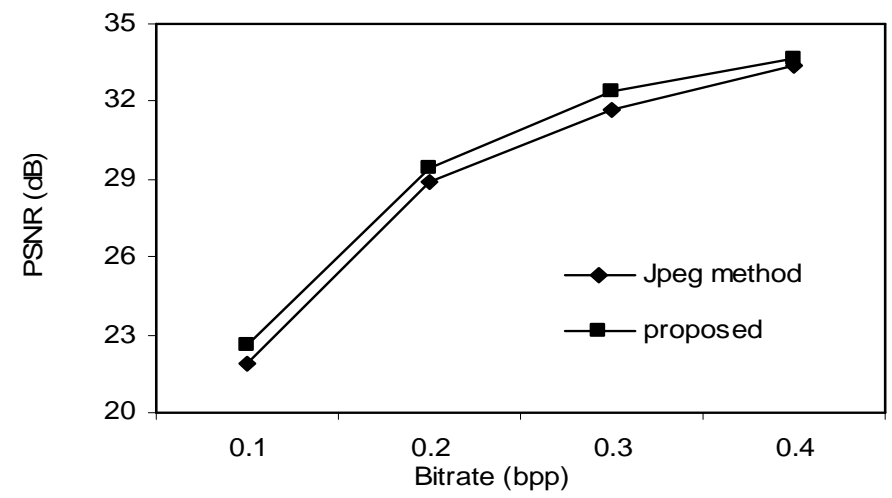

Fig.5 Comparison between the JPEG decoder results and the results of JPEG decoder after blocking artifact reductions for Lena image.

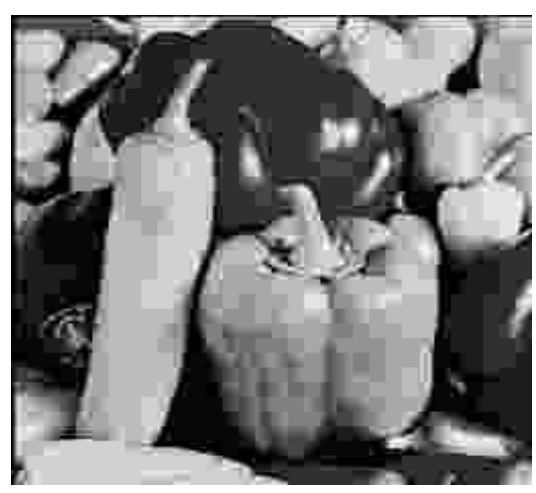

(a) With blocking artifacts

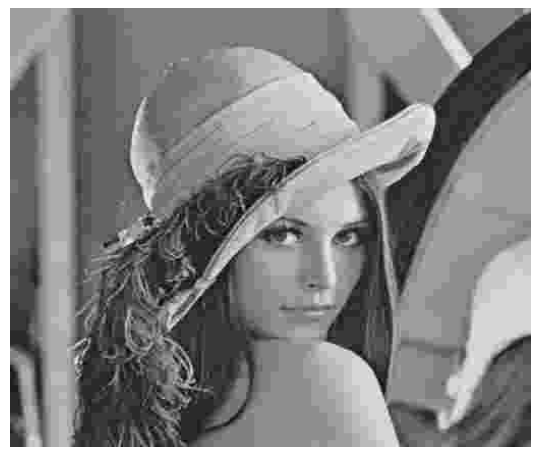

(a) With blocking artifacts

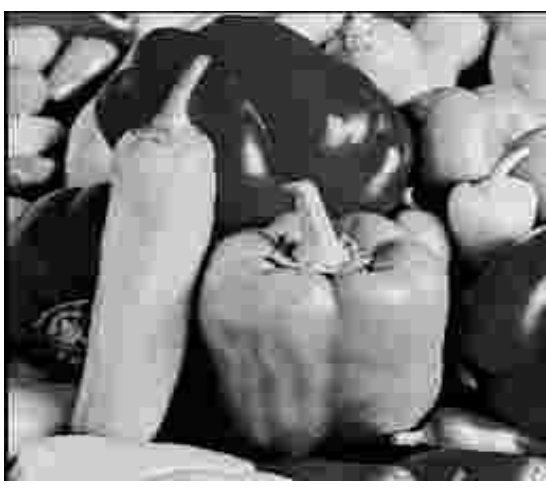

(b) After blocking artifact reduction

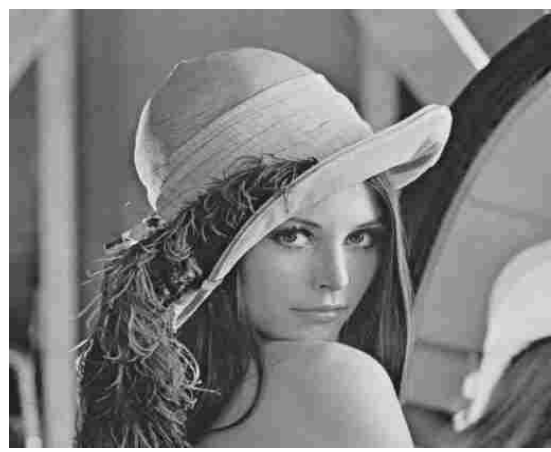

(b) After blocking artifact reduction

Fig. 6 The decoder output for both Peppers and Lena image at bitrate $0.2 \mathrm{bpp}$. 
Table 1 Comparison of PSNR for different post processing techniques

\begin{tabular}{|c|c|c|c|c|c|c|c|}
\hline Image & $\begin{array}{c}\text { Bit rate } \\
\text { (bpp) }\end{array}$ & JPEG & $\begin{array}{c}\text { Method } \\
{[30]}\end{array}$ & $\begin{array}{c}\text { Method } \\
{[31]}\end{array}$ & $\begin{array}{c}\text { Metho } \\
\mathrm{d}[33]\end{array}$ & $\begin{array}{c}\text { Method } \\
{[29]}\end{array}$ & $\begin{array}{c}\text { Proposed } \\
\text { method }\end{array}$ \\
\hline Boat & 0.247 & 31.14 & 31.67 & 31.02 & 30.05 & 31.14 & 31.33 \\
\hline Peppers & 0.169 & 27.13 & 27.86 & 27.01 & 27.69 & 28.02 & 27.94 \\
\hline
\end{tabular}

\section{CONCLUSIONS}

In this paper, a new method to blocking artifact detection and reduction is introduced. The key idea of the detection process is based on the power spectrum estimation of the e pixel absolute difference (PAD) in both horizontal and vertical directions. The power spectrum of the PAD is estimated using the Maximum Entropy method (MEM) which is a nonparametric method to perform accurate power spectrum estimation. The motivation for the MEM is that it is an intuitively satisfying approach to power spectrum density estimation in that a minimal amount of assumptions is made concerning the input data (PAD). The blocking artifact reduction process is based on the modified projection operator using the best matching pixel's vector in four blocks surrounding the boundary vector. Simulation results on JPEG decoder indicate that the proposed technique provides better PSNR and good perceptual quality of the decoded image.

\section{REFERENCES}

[1] H. Paek, R.-C. Kim, and S.-U. Lee, "On the POCS-based postprocessing technique to reduce the blocking artifacts in transform coded images," IEEE Trans. Circuits and Systems for Video Tech., vol.8, no.3, pp.358-367, June 1998.

[2] B. Ramamurthi and A. Gersho, "Nonlinear space-variant post-processing of block coded images," IEEE Trans. Acoust., Speech, Signal Processing, vol.34, pp.12581267, Oct. 1986.

[3] H. Reeve and J.S. Lim, "Reduction of blocking effects in image coding," Opt. Eng., vol.23, no.1, pp.34-37, Jan. 1984.

[4] Z. Wang, and D. Zhang, "A novel approach for the reduction of blocking effects in low-bit-rate image compression," IEEE Trans. Communications, vol.46, no.6, pp.732-734, June 1998.

[5] Y. Yang, and N.P. Galatsanos, "Removal of compression artifacts using projections onto convex sets and line process modeling," IEEE Trans. Image Processing, vol.6, no.10, pp.1345-1357, Oct. 1997.

[6] G.A. Triantafyllidis, D. Tzovaras, and M.G. Strintzis, "Blocking artifact detection and reduction in compressed data," IEEE Trans. Circuits and Systems for video Tech., vol.12, no.10, pp.877-890, Oct. 2002.

[7] B. Girod, "What's wrong with mean-squared error," Digital Images and Humman Vision, A.B. Watson, ed., pp.207-220, the MIT press, 1993.

[8] S.A. Karunasekera and N.G. Kingsbury, "A distortion measure for blocking artifacts in images based on human visual sensitivity," IEEE Trans. Image Processing, vol.4, no.6, pp.713-724, June 1995. 
[9] M. Miyahara, "Quality assessments for visual service," IEEE Communications Magazine, pp.51-60, Oct. 1988.

[10] H.S. Malvar and D.H. Staelin, "The LOT: Transform coding without blocking effects," IEEE Trans. Accoust., Speech, Signal Processing, vol.37, pp.553-559, 1989.

[11] H.S. Malvar, "Biorthogonal and non-uniform lapped transforms for transform coding with reduced blocking and ringing artifacts," IEEE Trans. Signal Processing, vol.46, pp.1043-1053, April 1998.

[12] J.G. Apostopoulos and N.S. Jayant, "Postprocessing for low bit-rate video compression," IEEE Trans. Image Processing, vol.8, pp.1125-1129, Aug. 1999.

[13] T. Jarske, P. Haavisto, and I. Defe'e, "Post-filtering methods for reducing blocking effects from coded images," IEEE Trans. Consumer Electron., pp.521526, Aug. 1994.

[14] T. Meier, K.N. Ngan, and G. Grebbin, "A region-based algorithm for enhancement of images degraded by blocking effects," in Proc. IEEE Tencon'96, vol.1, Perth, Australia, pp.405-408, Nov. 1996.

[15] Y.-F. Hsu and Y.-C. Chen, "A new adaptive separable median filter for removing blocking effects," IEEE Trans. Consumer Electron., pp.510-513, Aug. 1993.

[16] D.G. Sampson, D.V. Papadimitriou, and C. Chamzas, "Post-processing of blockcoded images at low bitrates," in Proc. 1996 IEEE Int. Conf. Image Processing, Lausanne, Switzerland, Sept. 1996.

[17] R. Molina, A.K. Katsaggelos, and J. Abad, "Bayesian image restoration using a wavelet-based subband decomposition," in Proc. 1999 Int. Conf. Acust., Speech, Signal Processing, Phoenix, AZ, Mar. 1999.

[18] Z. Xiong, M.T. Orchard, and Y.Q. Zhang, "A deblocking algorithm for JPEG Compressed images using overcomplete wavelet representations," IEEE Trans. Circuits and Systems for video Tech., vol.7, pp.692-695, Aug. 1999.

[19] N.C. Kim, I.H. Jang, D.H. Kim, and W.H. Hong, "Reduction of blocking artifact in block-coded images using wavelet transform," IEEE Trans. Circuits and Systems for video Tech., vol.8, pp.253-257, June 1998.

[20] H. Choi and T. Kim, "Blocking-artifact reduction in block-coded images using wavelet-based subband decomposition," IEEE Trans. Circuits and Systems for video Tech., vol.10, no.5, pp.801-805, Aug. 2000.

[21] Y. Yang, N.P. Galatsanos, and A.K. Katsaggelos, "Regularized reconstruction to reduce blocking artifacts of block discrete cosine transform compressed images," IEEE Trans. Circuits and Systems for video Tech., vol.3, pp.421-423, Dec. 1993.

[22] S. Minami and A. Zakhor, "An optimization approach for removing blocking effects in transform coding," IEEE Trans. Circuits and Systems for video Tech., vol.5, pp.74-82, April 1995.

[23] G. Lakhani and N. Zhong, "Derivation of prediction equations for blocking effect reduction," IEEE Trans. Circuits and Systems for video Tech., vol.9, pp.415-418, April 1999.

[24] W.B. Pennebaker and J.L. Mitchel, JPEG Still Image Data Compression Standard. New York:Van Nostrand, 1993.

[25] H.R. Wu and M. Yuen, "A generalized block-edge impairment metric for video coding," IEEE Signal Processing Letters, vol. 4, No.11, pp.317-320, Nov. 1997. 
[26] D. Wei and A.C. Bovik, "A new metric for blocking artifacts in block transformcoded images and video," Technical Report to Southwestern Bell Technology Resources, Inc. Oct. 1998.

[27] S.M. Kay, Modern Spectral Estimation: Theory and Application, Prentice Hall, Englewood Cliffs, NJ07632, 1988.

[28] D.Mulally and Hy. W. Paik," Power spectral density estimation simulation using the maximum entropy method, "in the proc. of the 36th Midwest Symposium on Circuits and Systems, vol. 1, pp. $578-581,1993$.

[29] S. Singh, V. Kumar, H.K. Verma, "Reduction of blocking artifacts in JPEG compressed images," journal of Digital Signal Processing, elsevier, vol. 17, pp. 225-243, 2007.

[30] Y.L. Lee, H.C. Kim, H.W. Park, "Blocking effect reduction of JPEG images by signal adaptive filtering," IEEE Trans. Image Process., vol.7, pp. 229-234, 1998.

[31] S. Liu, A.C. Bovik, "Efficient DCT-domain blind measurement and reduction of blocking artifacts," IEEE Trans. Circuits Syst. Video Technol., vol. 12, pp. 1139_ 1149, 2002.

[32] B. Zeng, "Reduction of blocking effect in DCT-coded images using zero-masking techniques," Signal Process., vol.79, 205-211, 1999.

[33] Y. Luo, R.K. Ward, "Removing the blocking artifacts of block-based DCT compressed images," IEEE Trans. Image Process., vol. 12, 838-842, 2003.

\section{تقتية تعمل على نقاط الصور المشفرة لتقليل التشوية بها نتيجة أستخدام التحويل فى عملية التثفير}

في هذا المقال تم تقديم طريقة جديدة لكثف وتقليل النشوبة فى الصور الناتج من ال blocking artifact ـ تتم عملية الكثف باستخدام استتناج ال power spectrum للقيمة المطلقة لفرق قيم نقط الصورة فى الأتجاه الأفقى وكذلك لأتجاه الرأسى للصورة. يتم استتناج ال power spectrum باستخدام طريقة أقصسى : تعطى نتائج جبده مع البيانات العشوائية التوزيع. و تتم عملية تقليل التشوبة باستخدام التماتل الذاتى لأستتناج أول

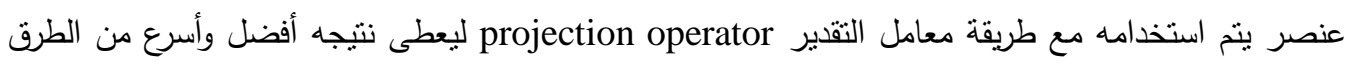
التقليديه. وأثبتت النتائج أن الطريقة المقدمة فى هذا البحث تعطى نتائج جيدة فى كثف وتثليل النتوبة في الصور

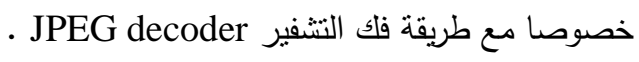

